\title{
Unintended target effect of anti-BCL-2 DNAi
}

This article was published in the following Dove Press journal:

Cancer Management and Research

22 September 2017

Number of times this article has been viewed

\author{
Abdul Shukkur Ebrahim' \\ Mustapha Kandouz ${ }^{2}$ \\ Nada Emara' \\ Amara B Sugalski ${ }^{3}$ \\ Leonard Lipovich ${ }^{3}$ \\ Ayad M Al-Katib' \\ 'Lymphoma Research Laboratory, \\ ${ }^{2}$ Department of Pathology, School \\ of Medicine, ${ }^{3}$ Center for Molecular \\ Medicine and Genetics, Wayne State \\ University, Detroit, MI, USA
}

Introduction: Previous research suggested that a novel compound PNT2258 inhibits B-cell lymphoma 2 (BCL-2) transcription by DNA interference (DNAi) and demonstrated its activity in preclinical xenograft models and in a pilot Phase II clinical trial in non-Hodgkin's lymphoma (NHL). While the drug downregulates $B C L-2$ at the promoter, mRNA, and protein levels, there is a significant homology (13-16 bases) between PNT100 and a number of promoters of genes involved in cell cycle regulation and survival. In this study, we identify cyclin-dependent kinase-4 (CDK4) as an unintended target gene of PNT2258 and examine its relevance to NHL. Methods: We performed a Basic Local Alignment Search Tool (BLAST) homology search using PNT100 DNAi sequences. Also, we conducted CDK4 promoter assay in K562 cells and studied the protein expression of CDK4 in Wayne State University (WSU)-follicular small cleaved cell lymphoma (FSCCL), WSU-diffuse large cell lymphoma, and WSU-Waldenström's macroglobulinemia (WM) lymphoma cells.

Results: BLAST homology search showed that PNT100 completely binds to BCL-2 gene as expected. However, there was 100\% homology in a stretch of 14 bases (8-21) between PNT100 and CDK4. PNT2258 strongly inhibited CDK4 promoter activity in K562 cells. Moreover, CDK4 protein expression was significantly downregulated by PNT2258 in WSU-FSCCL and WSU-WM cell lines.

Discussion: DNAi may work not only through knocking down the intended gene but also by knocking down other genes. PNT2258 affects $C D K 4$ expression and promoter activity. Results of the present study suggest a broader mechanism of action for DNAi targeting both intended (BCL-2) and unintended (CDK4) genes.

Keywords: non-Hodgkin's lymphoma, BCL-2, PNT2258, BLAST, CDK4

\section{Introduction}

Non-Hodgkin's lymphoma is the most common blood cancer in USA with $>72,000$ people estimated to be diagnosed in 2016; $>20,000$ will die of this disease. ${ }^{1}$ Our group has had a long-standing interest and track record in developing and evaluating different anti-B-cell lymphoma 2 (anti-BCL-2) strategies including small molecule inhibitors ${ }^{2-5}$ and antisense oligonucleotides. ${ }^{6,7}$ More recently, we have reported DNA interference (DNAi) as a new strategy for cancer therapy. ${ }^{8,9}$ DNAi represents nucleic acid based drugs that block transcription of specific genes involved in complex diseases such as cancer. PNT2258 is a BCL-2-targeting DNAi molecule which contains a single stranded sequence of 24 bases of unmodified phosphodiester DNA (known as PNT100) with a specific sequence, $5^{\prime}$-CACGCACGCGCATCCCCGCCCGTG-3'. PNT100 was designed to hybridize with genomic sequences that reside within the $5^{\prime}$-untranscribed
Correspondence: Ayad M Al-Katib Department of Internal Medicine, Lymphoma Research Laboratory, School of Medicine, Wayne State University, 8229 Scott Hall, 540 E Canfield, Detroit, MI 4820I, USA

$\mathrm{Tel}+\mathrm{I} 3135770982$

Fax + I 3/3 5770977

Email aal526@wayne.edu 
regulatory region of the $B C L-2$ gene to block its transcription via DNAi. The drug was evaluated in preclinical models of lymphoma and prostate cancer ${ }^{8}$ and has undergone a Phase I clinical trial. ${ }^{10}$

We recently demonstrated that PNT2258 significantly and dose-dependently inhibited $B C L-2$ at the promoter, RNA, and protein expression levels thus causing apoptosis. ${ }^{9}$ Expression of other $B C L-2$ family members, $B C L-x L, B A X, B A K$, and $B I D$, was unaffected. Interestingly, PNT2258 also induced S-phase arrest although $B C L-2$ is not involved in cell cycle regulation. This observation suggested that PNT2258 might silence other unintended genes (off-target effects). In fact, a Basic Local Alignment Search Tool (BLAST) search revealed that promoter sequences for a number of genes were partially homologous (13-16 out of 24 bases) with PNT100 sequences. In this report, we focus on cyclin-dependent kinase-4 (CDK4) gene as a primary unintended target given its roles in cell cycle regulation as a key regulator of the $\mathrm{G}_{1}-\mathrm{S}$ transition. We used three lymphoma cell lines with distinct genetic characteristics to investigate the unintended gene effect of PNT2258 and its role as DNAi. Our results show significant downregulation of $C D K 4$ promoter activity and protein expression following PNT2258 exposure in Wayne State University (WSU)-follicular small cleaved cell lymphoma (FSCCL) and WSU-Waldenström's macroglobulinemia (WM) cells.

\section{Methods and materials Cell culture}

The cell lines used in this study were WSU-FSCCL, WSUdiffuse large cell lymphoma (DLCL2), and WSU-WM, which were established in our laboratory at Wayne State University (WSU). ${ }^{1-13}$ WSU-FSCCL $(\mathrm{t}(14 ; 18)$ translocation for $B C L-2$ and $\mathrm{t}(8 ; 11) c-M Y C$ rearrangements), WSU-DLCL2 (t(14;18) $B C L-2$ rearrangement), and WSU-Waldenstrom's macroglobulinemia (WM; $\mathrm{t}(8 ; 14) c-M Y C$ rearrangement). All cell lines are Epstein-Barr virus negative and grow in liquid culture as previously described. ${ }^{14}$ The molecular characterization, translocations, and breakpoints of each cell line have been published. ${ }^{11}$ Human K562 cell line was obtained from American Type Culture Collection (ATCC, Manassas, VA, USA) and cells were maintained according to a standard protocol.

\section{Algorithm}

"BLAST 2 SEQUENCES" is an interactive tool that utilizes the BLAST engine for pairwise DNA-DNA or proteinprotein sequence comparison and is based on the same algorithm and statistics of local alignments that have been described earlier. ${ }^{15}$ The BLAST 2.0 algorithm generates a gapped alignment by using dynamic programming to extend the central pair of aligned residues. The heuristic methods confine the alignments to a predefined region of the path graph. A performance evaluation of the new gapped BLAST algorithm and its comparison to that of the original ungapped BLAST has been presented. ${ }^{16}$

\section{Luciferase assay}

The 404-bp fragment, containing the $C D K 4$ promoter subcloned into pGL3-basic luciferase expression vector (Promega, Madison, WI, USA), was a kind gift of Dr Gary L Firestone (Department of Molecular and Cell Biology, University of California at Berkeley, Berkeley, CA, USA). All transfections were carried out in 24-well plates. Briefly, plasmids ( $1 \mu \mathrm{g} C D K 4$ promoter construct, $0.1 \mu \mathrm{g}$ Renilla luciferase expressing reporter vector $\mathrm{pRL}-\mathrm{Null}$ [Promega]), were introduced into $<50 \%$ confluent K562 cells with Lipofectamine $_{\otimes}$ LTX transfection reagent (Life Technologies, Grand Island, NY, USA). The CDK4 and control empty vectors were transfected into K562 cells at a density of $0.2 \times 10^{6}$ viable cells $/ \mathrm{mL}$ per well in a 24 -well plate. Twentyfour hours after transfection, cells were treated with $2.5 \mu \mathrm{M}$ PNT2258 for 48 hours. Cells were lysed 48 hours later, and promoter activity analyzed in a MicroLumat Plus LB96V reader (Berthold Technologies, Bad Wildbad, Germany) using the Dual Luciferase ${ }_{\circledast}$ Reporter Assay System (Promega). The firefly luciferase values were normalized to those of Renilla luciferase; all transfections were repeated at least three times.

\section{Antibodies and reagents}

CDK4 antibody (DCS-35: sc-23896, 1:500) was purchased from Santa Cruz Biotechnology (Dallas, TX, USA) and the actin antibody (ACTN05, 1:2,000) from Thermo Fisher Scientific (Waltham, MA, USA). Protein concentrations were determined using the micro bicinchoninic acid protein assay (Pierce Chemical Company, Rockford, IL, USA). PNT2258 and associated reagents were provided by ProNAi Therapeutics (Plymouth, MI, USA). The drug PNT2258 consists of a 24 base unmodified DNA phosphodiester oligonucleotide encapsulated in amphoteric proprietary liposomes as previously described. ${ }^{8}$

\section{Immunoblotting}

PNT2258-treated and untreated cells were harvested, washed in phosphate-buffered saline, and lysed in M-PER lysis buffer containing a protease and phosphatase inhibitor cocktail (Thermo Fisher Scientific). ${ }^{9}$ Equal amounts of protein lysates were subjected to sodium dodecyl sulfate-polyacrylamide gel electrophoresis followed by blotting with the indicated antibod- 
ies and detection by Western SuperSignal West Pico Chemiluminescent Substrate reagents (Thermo Fisher Scientific). Select images were quantified using the ImageJ densitometry software (version 1.45; US National Institutes of Health, Bethesda, MD, USA) and normalized to the actin signal. Data are presented as relative band signal intensity compared to control.

\section{Data analysis and statistical significance}

Statistics were conducted using GraphPad Prism 5.0 for Windows (GraphPad Software, Inc, La Jolla, CA, USA) and tests were done with one-way analysis of variance $(* P<0.05$, $* * P<0.01, * * * P<0.001)$. $P$-values $<0.05$ were considered to be statistically significant. Results are displayed as averages with error bars indicating standard deviations. ImageJ (National Institute of Mental Health, Bethesda, MD, USA) densitometry software was used for quantification of Western blot bands. Selected bands were quantified based on their relative integrated intensities, calculated as the product of the selected pixel area and the mean gray value for those pixels normalized to the internal control.

\section{Results}

\section{BLAST search identifies multiple targets for the PNT2258 DNAi oligonucleotides}

The BLAST homology search showed that PNT100 does fully bind to the $B C L-2$ gene as predicted. However, portions of PNT100 sequence averaging 13-14 bases showed 100\% identity with four different genes: CDK4, insulin-like growth factor-1 receptor (IGF-1R), p21-activated kinase 3, and transferrin receptor (TFRC/CD71). As expected, CDK4, IGF$1 \mathrm{R}$, and TFRC are involved in cell cycle regulation. CDK4, IGF-1R, and TFRC may also modulate cell cycle/cell death via effects on cell metabolism. Specifically, there was $100 \%$ homology in 14 bases (8-21) between PNT100 and CDK4 (Figure 1) which is the subject of this report.

\section{PNT2258 represses the CDK4 promoter}

We have previously reported that the 24 bases PNT100 sequence included within PNT2258 is complementary to a segment of the consensus sequence recognized by the Sp1 transcription factor in the $B C L-2 \mathrm{P} 1$ promoter. ${ }^{9}$ To examine whether a putative region in the $C D K 4$ promoter confers PNT2258 responsiveness, we transiently transfected K562 cells with a plasmid containing a 404-bp long CDK4 promoter construct before exposure to PNT2258 for 48 hours. As shown in Figure 2, PNT2258 strongly downregulated $C D K 4$ promoter activity.

\section{PNT2258 downregulates CDK4 protein in lymphoma cells}

We examined whether PNT2258 inhibits $C D K 4$ expression in lymphoma cells. Western blot analysis revealed that CDK4

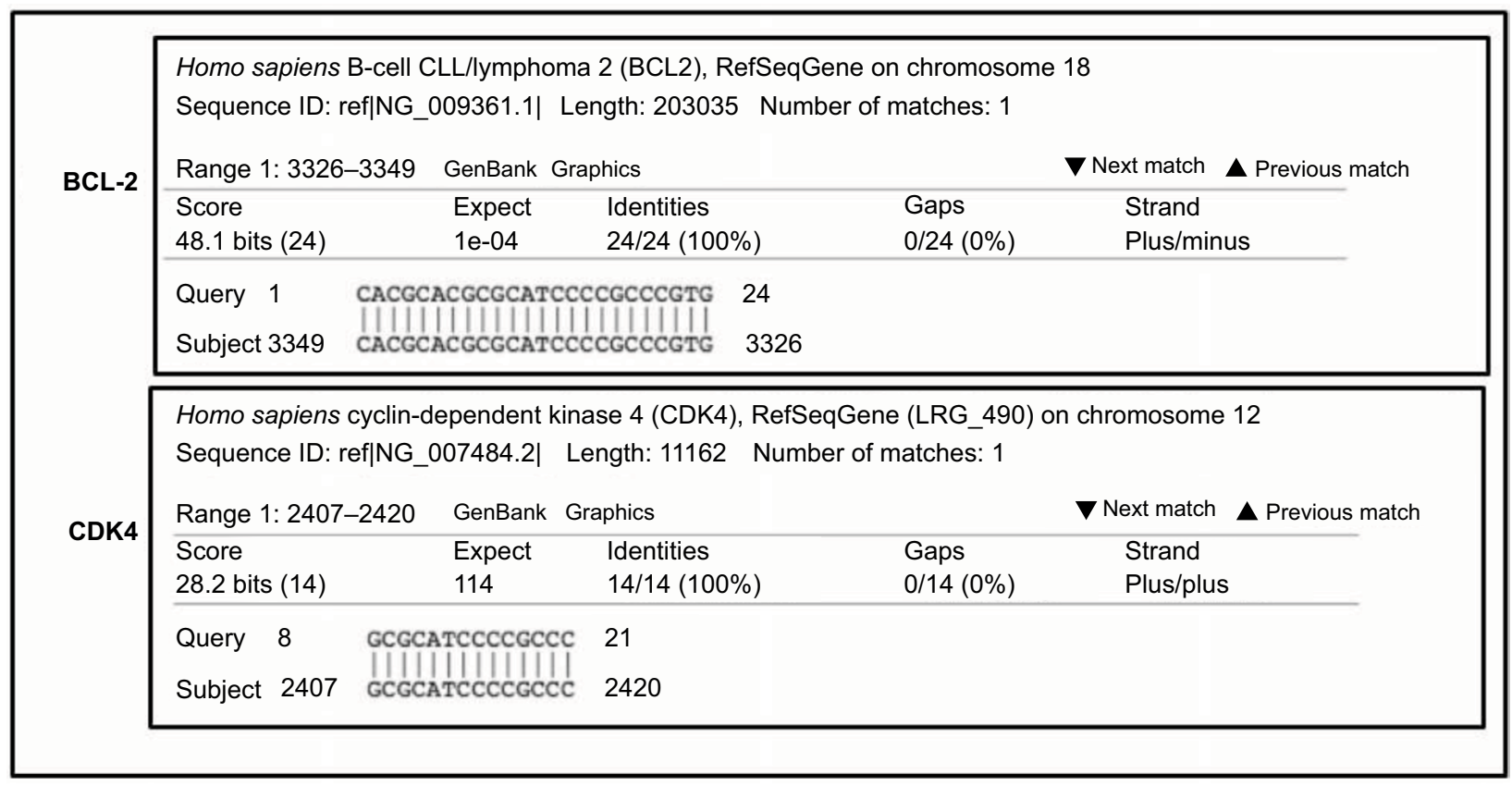

Figure I Significant homology between PNTI00 and CDK4.

Note: BLAST search homology between PNTI00 DNAi and CDK4 sequence (14 bases).

Abbreviations: BLAST, Basic Local Alignment Search Tool; DNAi, DNA interference; CDK4, cyclin-dependent kinase-4; BCL-2, B-cell lymphoma 2. 
protein levels were significantly decreased 24 through 72 hours following exposure to $2.5 \mu \mathrm{M}$ PNT2258 in WSUFSCCL and WSU-WM cells (Figure 3A and B). However, the CDK4 protein levels in WSU-DLCL2 cells were lower at 48-72 hours, but were not statistically significant (Figure 3C).

\section{Discussion}

WSU-FSCCL, WSU-DLCL2, and WSU-WM cells were used in a previous study as tools to investigate the effect of PNT2258 in vitro at different concentrations $(2.5,5.0$, and $10 \mu \mathrm{M}$ ) on cell growth and survival. PNT2258 induced apoptosis which can be explained by its effect on $B C L-2$ expression. ${ }^{9}$ A significant decrease in cell viability was observed after 48 hours in all of the cell lines exposed to PNT2258 at the lowest concentration $(2.5 \mu \mathrm{M}) .{ }^{9,17}$ Nevertheless, PNT2258 has additional effects on WSU-FSCCL cells, such as cell cycle arrest, that cannot be explained by $B C L-2$ targeting. ${ }^{18}$ Results of the present study suggest a broader mechanism of action for DNAi targeting both intended $(B C L-2)$ and unintended $(C D K 4)$ genes. Such an effect can enhance the DNAi anticancer effectiveness.

Our findings in this study have two implications: 1) PNT2258 inhibits the expression of CDK4 (which is one of the mechanisms of action of PNT2258 not previously

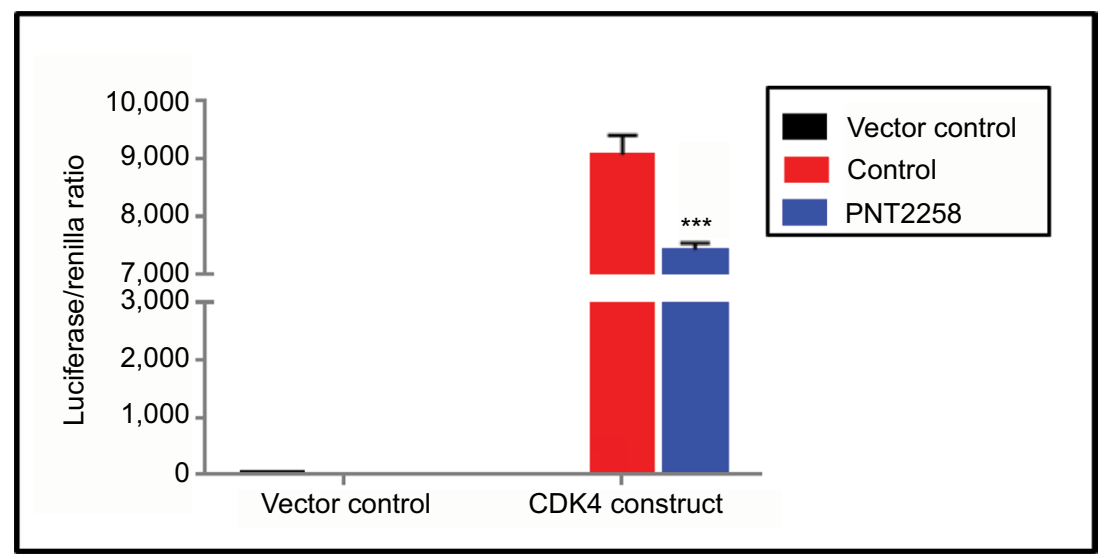

Figure 2 PNT2258 represses CDK4 promoter activity.

Notes: Luciferase activity of K562 cells transiently transfected with the CDK4 promoter construct was significantly lower in the presence of PNT2258. Results are representative of three independent experiments (***P<0.00I by ANOVA).

Abbreviations: ANOVA, analysis of variance; CDK4, cyclin-dependent kinase-4.

A

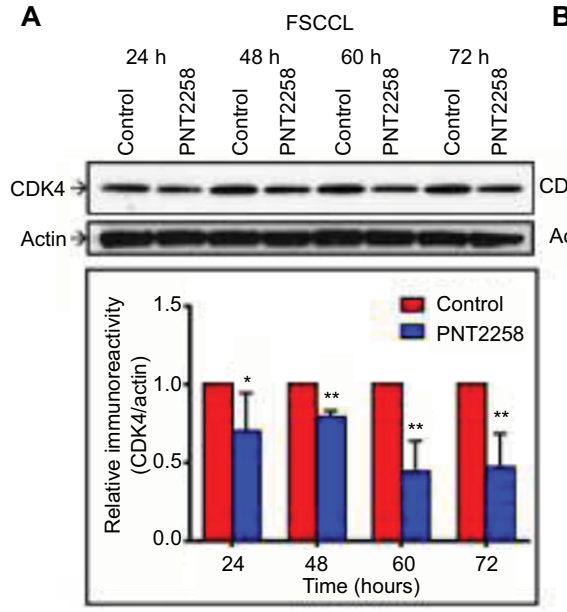

B
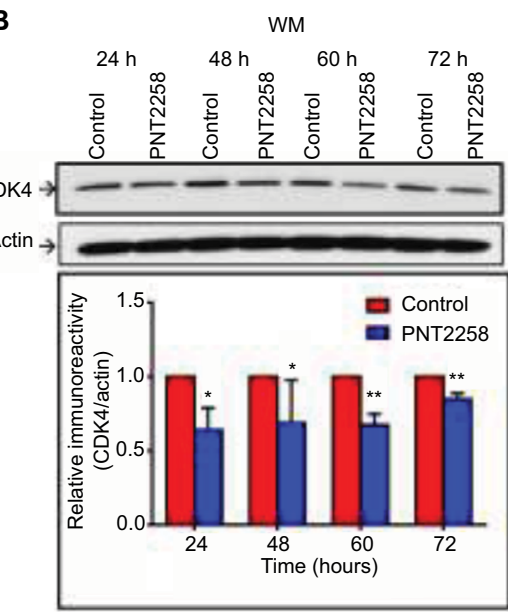

C

DLCL2

Figure 3 PNT2258 downregulates CDK4 protein expression in WSU-FSCCL and WSU-WM cells.

Notes: Representative Western blots demonstrating time-dependent decreases in CDK4 protein in PNT2258-treated cells; WSU-FSCCL (A), WSU-WM (B), and WSUDLCL2 (C). $\beta$-actin was used as loading control. Densitometric analysis of CDK4 band intensities (24-72 hours) normalized to $\beta$-actin. Results are representative of three independent experiments $(* P<0.05$ and $* * P<0.0$ I by ANOVA).

Abbreviations: ANOVA, analysis of variance; CDK4, cyclin-dependent kinase-4; WSU, Wayne State University; FSCCL, follicular small cleaved cell lymphoma; WM, Waldenström's macroglobulinemia; DLCL2, diffuse large cell lymphoma. 
recognized or predicted), and 2) decreased expression of CDK4 explains the previously observed effect of PNT2258 on cell cycle, a finding that cannot be explained by the effect of PNT2258 on the target gene (BCL-2).

Our previous findings suggest that BCL-2 family member MCL-1 protein levels were lower in PNT2258-treated cells. ${ }^{9}$ However, we believe that this decrease might be due to knocking down the unintended target gene CDK4, which may lead to a cell cycle arrest induced by PNT2258. MCL-1 downregulation was recently shown to be associated with $\mathrm{G}_{1} / \mathrm{S}$ transition. ${ }^{19}$ Moreover, it is not clear if complete 24 bases of DNAi against CDK4 will have a more significant knockdown of CDK4 expression compared with partial homology of PNT100 sequence. CDK4 has been a target for cancer therapy with recent US Food and Drug Administration approval of CDK4/6 inhibitors palbociclib (PD-0332991) and ribociclib (LEE011) for breast cancer. ${ }^{20,21}$

Inhibition of CDK4, IGF-1R, and TFRC leads to decreased proliferation and $\mathrm{G}_{1}$ cycle arrest. ${ }^{22-24}$ IGF-1R can regulate cell cycle progression at several steps by facilitating $\mathrm{G}_{0}-\mathrm{G}_{1}$ transition through $\mathrm{p} 70 \mathrm{~S} 6 \mathrm{~K}$ and promoting $\mathrm{G}_{1}-\mathrm{S}$ transition by increasing cyclin D1 and CDK4 gene expression. Since activation of IGF-1R and TFRC enhances reactive oxygen species, their inhibition improves mitochondrial function and lowers intrinsic apoptosis, reducing cytochrome c release and increasing caspase- 3 and poly(ADP-ribose) polymerase cleavage. ${ }^{22-24}$ These proapoptotic effects are consistent with similar effects of PNT2258 and, although awaiting experimental evidence, suggest that the latter's unintended effects on CDK4, IGF-1R, and TFRC are unlikely to result in harmful "off-target" effects.

Additional investigations are required to explore CDK4 targeting by DNAi as an alternative anti-CDK4 strategy. There remains the possibility of additional unintended target genes for PNT2258 that may also play a significant role as part of its broad mechanism of action.

\section{Conclusion}

In our study, we demonstrated that DNAi may work not only through knocking down their intended gene, but also by knocking down other genes. These possibilities should be kept in mind as new DNAi agents are developed.

\section{Abbreviations}

WSU: Wayne State University

NHL: non-Hodgkin's lymphoma

FSCCL: follicular small cleaved cell lymphoma
WM: Waldenström's macroglobulinemia

DLCL2: diffuse large cell lymphoma

DNAi: DNA interference

\section{Acknowledgments}

The authors wish to thank ProNAi Therapeutics for providing PNT2258 and related agents used in this study. This study was funded by a grant from ProNAi Therapeutics, by St John Hospital and Medical Center Foundation, and by Michigan Corporate Relations Network's Small Company Innovation Program. The data sets supporting the conclusions of this article are included within the article.

\section{Author contributions}

ASE designed and carried out all experiments, correlated and analyzed the resulting data, and organized and prepared the manuscript for publication. MK designed the promoter assay. NE provided technical assistance to ASE in carrying out experiments. LL and SBA provided guidance and analysis of BLAST search. AMA conceived and coordinated the overall project, participated in the design of experiments, and manuscript writing. All authors read and approved the final manuscript. All authors contributed toward data analysis, drafting and revising the paper and agree to be accountable for all aspects of the work.

\section{Disclosure}

The authors report no conflicts of interest in this work.

\section{References}

1. Siegel RL, Miller KD, Jemal A. Cancer statistics. CA Cancer J Clin. 2016;66(1):7-30.

2. Mohammad RM, Wang S, Aboukameel A, et al. Preclinical studies of a nonpeptidic small-molecule inhibitor of Bcl-2 and Bcl-X(L) [(-)-gossypol] against diffuse large cell lymphoma. Mol Cancer Ther. 2005;4(1):13-21.

3. Mohammad RM, Goustin AS, Aboukameel A, et al. Preclinical studies of TW-37, a new nonpeptidic small-molecule inhibitor of Bcl-2, in diffuse large cell lymphoma xenograft model reveal drug action on both Bcl-2 and Mcl-1. Clin Cancer Res. 2007;13(7):2226-2235.

4. Arnold AA, Aboukameel A, Chen J, et al. Preclinical studies of Apogossypolone: a new nonpeptidic pan small-molecule inhibitor of Bcl-2, Bcl-XL and Mcl-1 proteins in follicular small cleaved cell lymphoma model. Mol Cancer. 2008;7:20.

5. Al-Katib AM, Sun Y, Goustin AS, et al. SMI of Bcl-2 TW-37 is active across a spectrum of B-cell tumors irrespective of their proliferative and differentiation status. J Hematol Oncol. 2009;2:8.

6. Smith MR, AbubakrY, Mohammad R, et al. Antisense oligodeoxyribonucleotide down-regulation of bcl-2 gene expression inhibits growth of the low-grade non-Hodgkin's lymphoma cell line WSU-FSCCL. Cancer Gene Ther. 1995;2(3):207-212.

7. Mohammad R, Abubakr Y, Dan M, et al. Bcl-2 antisense oligonucleotides are effective against systemic but not central nervous system disease in severe combined immunodeficient mice bearing human t(14;18) follicular lymphoma. Clin Cancer Res. 2002;8(4):1277-1283. 
8. Rodrigueza WV, Woolliscroft MJ, Ebrahim AS, et al. Development and antitumor activity of a BCL-2 targeted single-stranded DNA oligonucleotide. Cancer Chemother Pharmacol. 2014;74(1):151-166.

9. Ebrahim AS, Kandouz M, Liddane A, et al. PNT2258, a novel deoxyribonucleic acid inhibitor, induces cell cycle arrest and apoptosis via a distinct mechanism of action: a new class of drug for non-Hodgkin's lymphoma. Oncotarget. 2016;7(27):42374-42384.

10. Tolcher AW, Rodrigueza WV, Rasco DW, et al. A phase 1 study of the BCL2-targeted deoxyribonucleic acid inhibitor (DNAi) PNT2258 in patients with advanced solid tumors. Cancer Chemother Pharmacol. 2014;73(2):363-371.

11. Mohammad RM, Mohamed AN, Smith MR, Jawadi NS, al-Katib A. A unique EBV-negative low-grade lymphoma line (WSU-FSCCL) exhibiting both $\mathrm{t}(14 ; 18)$ and $\mathrm{t}(8 ; 11)$. Cancer Genet Cytogenet. 1993;70(1): $62-67$.

12. Al-Katib AM, Smith MR, Kamanda WS, et al. Bryostatin 1 downregulates $\mathrm{mdr} 1$ and potentiates vincristine cytotoxicity in diffuse large cell lymphoma xenografts. Clin Cancer Res. 1998;4(5):1305-1314.

13. al-Katib A, Mohammad R, Hamdan M, Mohamed AN, Dan M, Smith MR. Propagation of Waldenstrom's macroglobulinemia cells in vitro and in severe combined immune deficient mice: utility as a preclinical drug screening model. Blood. 1993;81(11):3034-3042.

14. Al-Katib AM, Aboukameel A, Mohammad R, Bissery MC, Zuany-Amorim C. Superior antitumor activity of SAR3419 to rituximab in xenograft models for non-Hodgkin's lymphoma. Clin Cancer Res. 2009;15(12): 4038-4045.
15. Altschul SF, Madden TL, Schäffer AA, et al. Gapped BLAST and PSIBLAST: a new generation of protein database search programs. Nucleic Acids Res. 1997;25(17):3389-3402.

16. Altschul SF, Gish W, Miller W, Myers EW, Lipman DJ. Basic local alignment search tool. J Mol Biol. 1990;215(3):403-410.

17. Ebrahim AS, Sabbagh H, Liddane A, Raufi A, Kandouz M, Al-Katib A. Hematologic malignancies: newer strategies to counter the BCL-2 protein. J Cancer Res Clin Oncol. 2016;142(9):2013-2022.

18. Moldoveanu T, Follis AV, Kriwacki RW, Green DR. Many players in BCL-2 family affairs. Trends Biochem Sci. 2014;39(3):101-111.

19. Morciano G, Giorgi C, Balestra D, et al. Mcl-1 involvement in mitochondrial dynamics is associated with apoptotic cell death. Mol Biol Cell. 2016;27(1):20-34.

20. O'Leary B, Finn RS, Turner NC. Treating cancer with selective CDK4/6 inhibitors. Nat Rev Clin Oncol. 2016;13(7):417-430.

21. Finn RS, Aleshin A, Slamon DJ. Targeting the cyclin-dependent kinases (CDK) 4/6 in estrogen receptor-positive breast cancers. Breast Cancer Res. 2016;18(1):17.

22. Hamilton E, Infante JR. Targeting CDK4/6 in patients with cancer. Cancer Treat Rev. 2016;45:129-138.

23. Samani AA, Yakar S, LeRoith D, Brodt P. The role of the IGF system in cancer growth and metastasis: overview and recent insights. Endocr Rev. 2007;28(1):20-47.

24. O’Donnell KA, Yu D, Zeller KI, et al. Activation of transferrin receptor 1 by c-Myc enhances cellular proliferation and tumorigenesis. $\mathrm{Mol} \mathrm{Cell}$ Biol. 2006;26(6):2373-2386.
Cancer Management and Research

\section{Publish your work in this journal}

Cancer Management and Research is an international, peer-reviewed open access journal focusing on cancer research and the optimal use of preventative and integrated treatment interventions to achieve improved outcomes, enhanced survival and quality of life for the cancer patient. The manuscript management system is completely online and includes

\section{Dovepress}

a very quick and fair peer-review system, which is all easy to use. Visit http://www.dovepress.com/testimonials.php to read real quotes from published authors. 